\title{
Inertial- and Dissipation-Range Asymptotics in Fluid Turbulence
}

\author{
Sujan K. Dhar, Anirban Sain, and Rahul Pandit* \\ Department of Physics, Indian Institute of Science, Bangalore 560 012, India
}

\begin{abstract}
We propose and verify a wave-vector-space version of generalized extended self-similarity [R. Benzi et al., Europhys. Lett. 32, 709 (1995)] and broaden its applicability to uncover intriguing, universal scaling in the far dissipation range by computing high-order $(\leq 20)$ structure functions numerically for (1) the three-dimensional, incompressible Navier-Stokes equation (with and without hyperviscosity) and (2) the Gledzer-Ohkitani-Yamada shell model for turbulence. Also, in case (2), with Taylor-microscale Reynolds numbers $4 \times 10^{4} \leq \mathrm{Re}_{\lambda} \leq 3 \times 10^{6}$, we find that the inertial-range exponents $\left(\zeta_{p}\right)$ of the order- $p$ structure functions do not approach their Kolmogorov value $p / 3$ as $\operatorname{Re}_{\lambda}$ increases. [S0031-9007(97)02862-7]
\end{abstract}

Kolmogorov's pioneering work (K41) [1] on homogeneous isotropic turbulence used the cascade picture to predict simple scaling forms for velocity structure functions (see below). These forms hold for distances $r$ in the inertial range that lies between $L$, the forcing scale, and $\eta_{d}$, the dissipation scale at which viscosity starts modifying the invariant energy cascade. Subsequent studies [2-11] have refined K41, as we outline below, but have concentrated principally on the inertial range. In this Letter we use recently developed generalizations of such scaling $[2,5]$ to elucidate the crossover from inertial- to dissipation-range behaviors in fluid turbulence.

The order- $p$ velocity structure functions $S_{p}(r) \equiv$ $\left\langle\left|\mathbf{v}_{i}(\mathbf{x}+\mathbf{r})-\mathbf{v}_{i}(\mathbf{x})\right|^{p}\right\rangle$, where $i(=1,2$, or 3$)$ denotes components, scale as $S_{p}(r) \sim r^{\zeta_{p}}$ at high Reynolds numbers $\operatorname{Re}_{\lambda}$ and for the inertial range $20 \eta_{d} \lesssim r \ll L$ (where $\lambda$ is the Taylor microscale). The K41 result $\zeta_{p}=p / 3$ works well for $p \lesssim 4$; but for large $p$, most studies [2-11] find multiscaling, i.e., $\zeta_{p}=p / 3-\delta \zeta_{p}$, a nonlinear increasing function with $\delta \zeta_{p}>0$. Also, a procedure called extended self-similarity (ESS) [5], in which $\zeta_{p}$ is obtained from $S_{p} \sim S_{3}^{\zeta_{p}}$, extends the apparent inertial range down to $r \simeq 5 \eta_{d}$. A more recent technique, generalized extended self-similarity (GESS) [2], uses the dimensionless structure functions $G_{p}(r) \equiv S_{p}(r) /\left[S_{3}(r)\right]^{p / 3}$ and suggests that the form $G_{p} \sim\left[G_{q}\right]^{\rho_{p q}}$, with $\rho_{p, q}=\left[\zeta_{p}-p \zeta_{3} / 3\right] /\left[\zeta_{q}-q \zeta_{3} / 3\right]$, holds down to the lowest resolvable values of $r$. GESS has been tested [2] to some extent $(p, q \leq 6)$. We show that ESS and GESS provide us with sensitive ways of studying the crossover of structure functions from their inertial- to dissipation-range forms.

Specifically, we show how GESS is modified at sufficiently small $r$ by computing wave-vector-space ( $k$ space) analogs of high-order $(\leq 20)$ structure functions for (1) the three-dimensional, incompressible Navier Stokes equation (3D NS), with and without hyperviscosity, and (2) the Gledzer-Ohkitani-Yamada (GOY) shell model for turbulence [9-12] (where we attain both large $\operatorname{Re}_{\lambda}$ and $k \gg k_{d} \equiv \eta_{d}^{-1}$ ). We further propose a $k$-space GESS
[2], show that it holds for $L^{-1} \ll k \lesssim 1.5 k_{d}$, but then crosses over to another form in the far dissipation range. To study this we postulate $k$-space ESS [for real-space structure functions we use the symbols $S$ and $G$ and for their $k$-space analogs (not Fourier transforms) the symbols $S$ and $G]$ :

$$
\begin{aligned}
& S_{p} \equiv\left\langle|\mathbf{v}(\mathbf{k})|^{p}\right\rangle \approx A_{I p}\left(S_{3}\right)^{\zeta_{p}^{\prime}}, \quad L^{-1} \ll k \lesssim 1.5 k_{d}, \\
& S_{p} \equiv\left\langle|\mathbf{v}(\mathbf{k})|^{p}\right\rangle \approx A_{D p}\left(S_{3}\right)^{\alpha_{p}}, \quad 1.5 k_{d} \lesssim k \ll \Lambda,(1)
\end{aligned}
$$

where $A_{I p}$ and $A_{D p}$ are, respectively, nonuniversal amplitudes for inertial and dissipation ranges and $\Lambda^{-1}$ the (molecular) length at which hydrodynamics fails (see [5,6] for real-space analogs). Our study shows (Figs. 1 and 2)

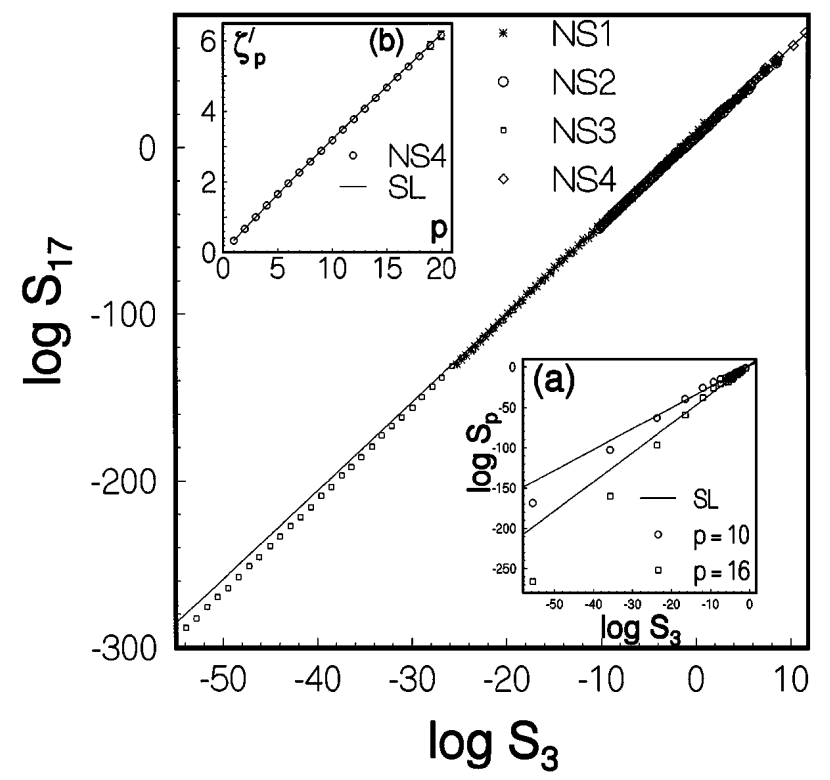

FIG. 1. Log-log plots (base 10) of $S_{p}$ versus $S_{3}$ for 3D NS ( $p=17$ for runs NS1-4) and GOY [run G1 in inset (a)] models showing our $k$-space ESS [Eq. (1)]; full lines are the SL prediction [4]. Inset (b): $\zeta_{p}^{\prime}$ (circles) from run NS4; the line is $\zeta_{p}^{\prime}=2\left(\zeta_{p}+3 p / 2\right) / 11$, with the $\zeta_{p}=\zeta_{p}^{\mathrm{SL}}$. Note the deviation of our data points from SL lines at small $S_{3}$, i.e., in the dissipation range; this shows clearly only for NS3 on this scale, but is also present in runs NS1 and NS2 (Fig. 3). 


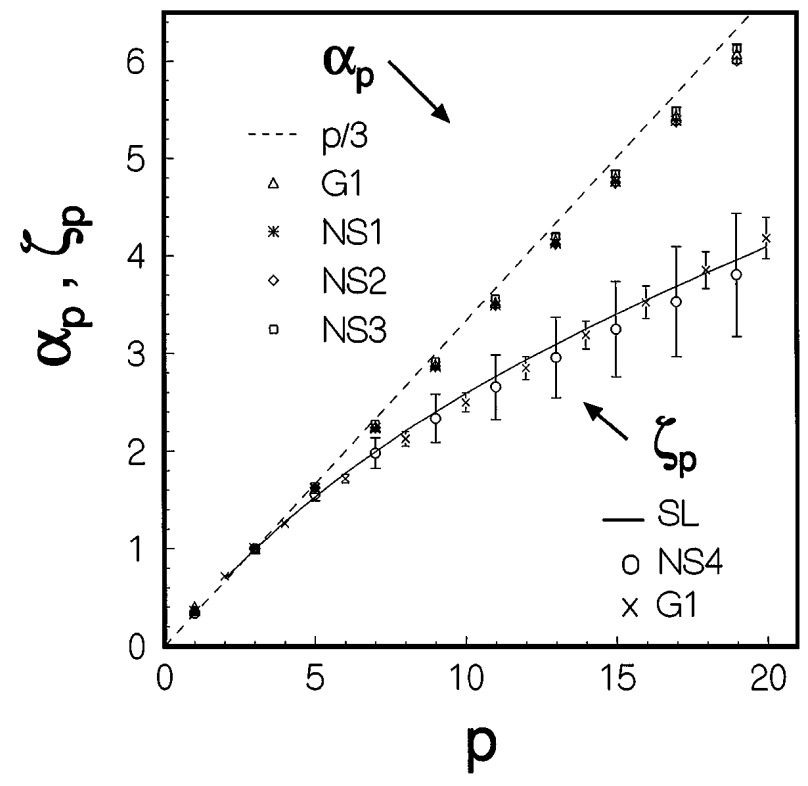

FIG. 2. Inertial- and dissipation-range exponents $\zeta_{p}$ and $\alpha_{p}$ (extracted from plots like Fig. 1) versus $p$ for GOY and NS runs and their comparison with the SL formula [4] and $p / 3$. We obtain $\zeta_{p}$ from our measured $\zeta_{p}^{\prime}$ and the formula $\zeta_{p}=11 \zeta_{p}^{\prime} / 2-3 p / 2$; this amplifies the error bars relative to Fig. 1(b). Error bars for $\alpha_{p}$ are shown but not apparent since they are comparable to the symbol sizes.

that Eq. (1) holds with two different exponents $\alpha_{p}$ and $\zeta_{p}^{\prime}$. In the GOY model $\zeta_{p}^{\prime}=\zeta_{p}$, but we find explicitly [Fig. 1(b)] that, for the 3D NS case, $\zeta_{p}^{\prime}=2\left(\zeta_{p}+\right.$ $3 p / 2) / 11$ [i.e., $S_{p}(k) \sim k^{-\left(\zeta_{p}+3 p / 2\right)}$ in the inertial range [13]]; the difference between the two arises because of phase-space factors. Both $\zeta_{p}$ and $\alpha_{p}$ (Fig. 2) seem universal [the same for all GOY and 3D NS runs (Table I) [14]]. $\zeta_{p}$ agrees fairly with the She-Leveque (SL) [4] formula $\zeta_{p}^{\mathrm{SL}}=p / 9+2\left[1-(2 / 3)^{p / 3}\right]$ for the ranges of $p$ and $\operatorname{Re}_{\lambda}$ in Fig. 2; and $\alpha_{p}$ is close to, but systematically less than, $p / 3$. The $k$ dependences of the inertial- and dissipation-range asymptotic behaviors follow now from the dependence of $S_{3}$ on $k$ : We find

$$
\begin{gathered}
S_{3} \approx B_{I} k^{-\zeta_{3}-9 / 2}, \quad L^{-1} \ll k \lesssim 1.5 k_{d}, \\
S_{3} \approx B_{D} k^{\delta} \exp \left(-c k / k_{d}\right), \quad 1.5 k_{d} \lesssim k \ll \Lambda,
\end{gathered}
$$

where $B_{I}$ and $B_{D}$ are, respectively, nonuniversal amplitudes [Eq. (2) holds [13] for 3D NS; for GOY the factor $9 / 2$ is absent]. Thus, in the far dissipation range, all $S_{p} \sim k^{\theta_{p}} \exp \left(-c \alpha_{p} k / k_{d}\right)$ for $1.5 k_{d} \lesssim k \ll \Lambda$, with $\theta_{p}=\alpha_{p} \delta$, a form not easy to verify numerically for large $p$, given the rapid decay at large $k$, and suggested hitherto [15] only for $S_{2}$. In Eq. (3), $\delta, c, k_{d}$ are not universal, but we extract the universal part of the crossover via our $k$-space GESS: Define $G_{p} \equiv S_{p} /\left(S_{3}\right)^{p / 3}$; log$\log$ plots of $G_{p}$ versus $G_{q}$ yield curves [Figs. 3(a) and 3(b)] with asymptotes which have universal, but different, slopes in inertial and dissipation ranges. The inertialrange asymptote has a slope $\rho(p, q)$ (as in real-space
GESS [2] which follows from the formulas above); the resulting $\zeta_{p}$ are in fair agreement with the SL formula [4]. The dissipation-range asymptote has a slope $\omega(p, q) \equiv$ $\left[\alpha_{p}-p / 3\right] /\left[\alpha_{q}-q / 3\right]$. The slopes of these asymptotes are universal, but the point at which the curve veers off from the inertial-range asymptote depends on the model (GOY, NS, etc.). However, a simple transformation yields a universal crossover scaling function [different for each $(p, q)$ pair because of multiscaling]: Define $\log _{10}\left(H_{p q}\right) \equiv D_{p q} \log _{10}\left(G_{p}\right)$ and $\log _{10}\left(H_{q p}\right) \equiv$ $D_{q p} \log _{10}\left(G_{q}\right)$; the scale factors $D_{p q}=D_{q p}$ are nonuniversal, but plots of $\log _{10}\left(H_{p q}\right)$ versus $\log _{10}\left(H_{q p}\right)$ show data from all GOY and 3D NS runs collapsing onto one universal curve within our error bars [Fig. 3(c) for $p=6$ and $q=9$ ] for all $k$ and $\operatorname{Re}_{\lambda}$. (This transformation holds the G1-8 GESS plots fixed and stretches the NS plots, without changing their slopes, until the asymptotes match.) Both ESS (Fig. 1) and GESS (Fig. 3) remove the exponential controlling factor [16] from the leading asymptotic behavior of $S_{p}$ in the far dissipation range and expose the remaining power-law dependence on $k$. Also, it is easy to see analytically that GESS plots (Fig. 3) amplify slope differences between inertial- and dissipationrange asymptotes relative to ESS plots (Fig. 1).

How robust is the fair agreement of $\zeta_{p}$ (Fig. 2) with the SL formula? Some studies [17-19] suggest that, as $\operatorname{Re}_{\lambda} \rightarrow \infty, \delta \zeta_{p} \equiv\left(p / 3-\zeta_{p}\right) \rightarrow 0$. Numerical solutions of the 3D NS equation can at best achieve [7-20] $\operatorname{Re}_{\lambda} \lesssim 220$, too small, by far, to resolve this issue, so we address it for the GOY model by studying the range $4 \times 10^{4} \lesssim \operatorname{Re}_{\lambda} \lesssim 3 \times 10^{6}$. We find (Fig. 4) that $\delta \zeta_{p}$ does not vanish with increasing $\operatorname{Re}_{\lambda}$, but rises marginally [21]. Systematic experiments at high $\operatorname{Re}_{\lambda}$ can check if the trends of Fig. 4 obtain in the NS case.

We remark that if we assume the hierarchy $\left[G_{p+1} / G_{p}\right]=\left[G_{p} / G_{p-1}\right]^{\gamma}\left[\lim _{p \rightarrow \infty} G_{p+1} / G_{p}\right]^{1-\gamma}$ with $\gamma^{3}=2 / 3$ (whose real-space analog is equivalent [2] to the SL moment hierarchy for the energy dissipation [4]) and use [22] $G_{p}(k) \approx C_{p} k^{\beta_{p}}$, we get a difference equation for $\beta_{p}$ identical to the SL one (our $\beta_{p}$ is their $\left.-\tau_{p / 3}\right)$. This, when solved with the boundary conditions $\beta_{0}=\beta_{3}=0$ and $\lim _{p \rightarrow \infty}\left(\beta_{p+1}-\beta_{p}\right)=2 / 9$, yields the SL formula (via $\zeta_{p}=-\beta_{p}+p \zeta_{3} / 3$ ). However, our GESS yields $\left[G_{p+1} / G_{p}\right] \approx C_{p}^{\prime}\left[G_{p} / G_{p-1}\right]^{\Upsilon_{p}}$ with $\Upsilon_{p}=\left(\zeta_{p+1}-\zeta_{p}-1 / 3\right) /\left(\zeta_{p}-\zeta_{p-1}-1 / 3\right)$. Superficially, this might seem to violate the hierarchy assumed above, but it turns out to be consistent with our GESS form, if $Y_{p}=\gamma-2(1-\gamma) /\left[9\left(\zeta_{p}-\zeta_{p-1}-\zeta_{3} / 3\right)\right]$, which is precisely the SL difference equation. Of course, our GESS form can hold with $\zeta_{p} \neq \zeta_{p}^{\mathrm{SL}}$; Fig. 2 shows the quality of agreement between our measured $\zeta_{p}$ and $\zeta_{p}^{\mathrm{SL}}$.

We use a pseudospectral method [7] to solve the incompressible 3D NS equation. We force the first two $k$ shells, use a box with side $L_{B}=\pi$ and $64^{3}$ modes. Our dissipation term $-\left(\nu+\nu_{H} k^{2}\right) k^{2}$ allows for both viscosity $\nu$ and hyperviscosity $\nu_{H}$. For time integration 
TABLE I. Parameters $\nu$ (viscosity), $\nu_{H}$ (hyperviscosity), $\operatorname{Re}_{\lambda}$ (Taylor-microscale Reynolds number), $\tau_{e}$ (box-size eddy-turnover time), $\tau_{\text {av }}$ (averaging time), $\tau_{t}$ (transient time), and $k_{d}$ (dissipation-scale wave number) for our 3D NS runs NS1-4 ( $\left.k_{\max }=64\right)$ and GOY-model runs G1-8 $\left(k_{\max }=2^{22} k_{0}\right)$. The step size $(\delta t)$ used is 0.02 for NS1-4, $10^{-4}$ for G1-4, and $2 \times 10^{-5}$ for G5-8. Note $\tau_{e} \simeq 8 \tau_{I}$, the integral time for our NS runs.

\begin{tabular}{|c|c|c|c|c|c|c|c|}
\hline Run & $\nu$ & $\nu_{H}$ & $\operatorname{Re}_{\lambda}$ & $\tau_{e} / \delta t$ & $\tau_{t} / \tau_{e}$ & $\tau_{\mathrm{av}} / \tau_{e}$ & $k_{\max } / k_{d}$ \\
\hline $\mathrm{NS} 2$ & $2 \times 10^{-4}$ & 0 & $\simeq 8$ & $\simeq 3 \times 10^{4}$ & $\simeq 1$ & $\simeq 2.5$ & $\simeq 2.3$ \\
\hline NS4 & $5 \times 10^{-4}$ & $10^{-6}$ & $\simeq 22$ & $\simeq 3 \times 10^{3}$ & $\simeq 10$ & $\simeq 7$ & $\simeq 2$ \\
\hline G1-4 & $5 \times 10^{-6}-10^{-7}$ & 0 & $4 \times 10^{4}-3 \times 10^{5}$ & $\simeq(1.5-2.0) \times 10^{4}$ & $\simeq 500$ & $\simeq 2500$ & $\simeq 2^{5}-2^{3}$ \\
\hline G5-8 & $5 \times 10^{-8}-10^{-9}$ & 0 & $3.5 \times 10^{5}-3 \times 10^{6}$ & $\simeq(0.7-1) \times 10^{5}$ & $\simeq 500$ & $\simeq 2500$ & $\simeq 2^{3}-1$ \\
\hline
\end{tabular}

we use an Adams-Bashforth scheme (step size $\delta t$ ) [7]. Parameters for our 3D NS runs NS1-4 are given in Table I, where $\tau_{e} \equiv L_{B} / v_{\mathrm{rms}}$ is the box-size eddyturnover time and $\tau_{\mathrm{av}}$ the averaging time, after initial transients have decayed over a period $\tau_{t}$. We use $\operatorname{Re}_{\lambda}=$ $v_{\mathrm{rms}} \lambda / \nu$, where $\lambda=\left[\int_{0}^{\infty} E(k) d k / \int_{0}^{\infty} k^{2} E(k) d k\right]^{1 / 2}$, $v_{\mathrm{rms}}=\left[\left(2 / 3 L_{B}^{3}\right) \int_{0}^{\infty} E(k) d k\right]^{1 / 2}$, and $E(k) \sim S_{2}(k) k^{2}$. All $S_{p}(k)$ are averaged over shells of radius $k$. Care must be exercised in choosing $\delta t$ and the forcing amplitude, otherwise there is a slow but systematic stretching of the points along the asymptotes in Figs. 1 and 3 with increasing $\tau_{\mathrm{av}}$ (over the time scales of our low- $\mathrm{Re}_{\lambda} \mathrm{NS}$ runs). Fortunately, this hardly affects our exponents: Any attendant systematic errors in Fig. 2 are certainly less than the random errors indicated. All our NS runs use quadruple-precision arithmetic and we have checked that halving our integration time step does not affect our results perceptibly. Note also that sample fluctuations over even a few orders of magnitude are unimportant, given the range of our log-log plots like Fig. 1. Also, the agreement between our GOY and NS runs confirms our results. Our GOY-model data are, of course, of much better quality. Here Fourier components of the velocity are labeled by a discrete set of wave vectors $k_{n}=k_{0} q^{n}$. The dynamical variables are the complex, scalar velocities $v_{n}$ for each shell $n ; v_{n}$ is affected directly only by the velocities in nearest and next-nearest shells. This model yields scaling properties [9-12] akin to experimental ones. The GOY-model equations are

$$
\frac{d}{d t} v_{n}=i C_{n}-\nu k_{n}^{2} v_{n}+f_{n},
$$

where $\nu$ is the kinematic viscosity, $f_{n}$ the external force on shell $n, \quad C_{n}=\left(a k_{n} v_{n+1} v_{n+2}+\right.$ $\left.b k_{n-1} v_{n-1} v_{n+1}+c k_{n-2} v_{n-1} v_{n-2}\right)^{*}, \quad$ and $a, b$, and $c$ can be fixed up to a constant by demanding [11], for $\nu, f_{n}=0$, that $v_{n} \sim k_{n}^{-1 / 3}$ be a stationary solution of Eq. (4), and the GOY-model kinetic energy and helicity be conserved. We adopt the conventional parameters $[10,11] k_{0}=2^{-4}, \quad q=2, \quad a=1$, $b=c=-1 / 2$, and use $f_{n}=5 \times 10^{-3}(1+i) \delta_{n, 1}$, i.e., we force the first shell [23]. The GOY-model structure functions are $S_{n, p} \equiv\left\langle\left|v_{n}\right|^{p}\right\rangle \sim k_{n}^{-\zeta p} \quad[9-$ 11]; reliable values of $\zeta_{p}$ obtain [11] if we use $\Sigma_{n, p}=\left\langle\left|\operatorname{Im}\left[\boldsymbol{v}_{n} \boldsymbol{v}_{n+1} \boldsymbol{v}_{n+2}+\boldsymbol{v}_{n-1} \boldsymbol{v}_{n} \boldsymbol{v}_{n+1} / 4\right]\right|^{p / 3}\right\rangle \quad$ since this removes an underlying three cycle. We have used $\Sigma_{n, p}$ to obtain Fig. 4 [24], but $S_{n, p}$ in Figs. 1-3 for consistency with 3D NS. We use an Adams-Bashforth scheme [10] (step size $\delta t$ ) to integrate Eq. (4). The average of the time scale associated with the smallest wave number $\left(\left|v_{1}\right| k_{1}\right)^{-1}$ gives the "box-size" eddy turnover time. Table I lists other parameters for our 8 GOY-model runs G1-8, for which we use (cf. [10]) $E(k)=S_{n, 2} / k_{n}, \lambda=2 \pi / k_{0}\left[\Sigma_{n} S_{n, 2} / \Sigma_{n} k_{n}^{2} S_{n, 2}\right]^{1 / 2}$, and $v_{\mathrm{rms}}=\left[k_{0} \Sigma_{n} S_{n, 2} / \pi\right]^{1 / 2}$. This yields $\operatorname{Re}_{\lambda} \sim \nu^{-0.5}$, as expected [25] at large $\operatorname{Re}_{\lambda}$. Our GOY model runs are done using double-precision arithmetic, but we have repeated run $\mathrm{Gl}$ in quadruple precision and checked that our results are unchanged.
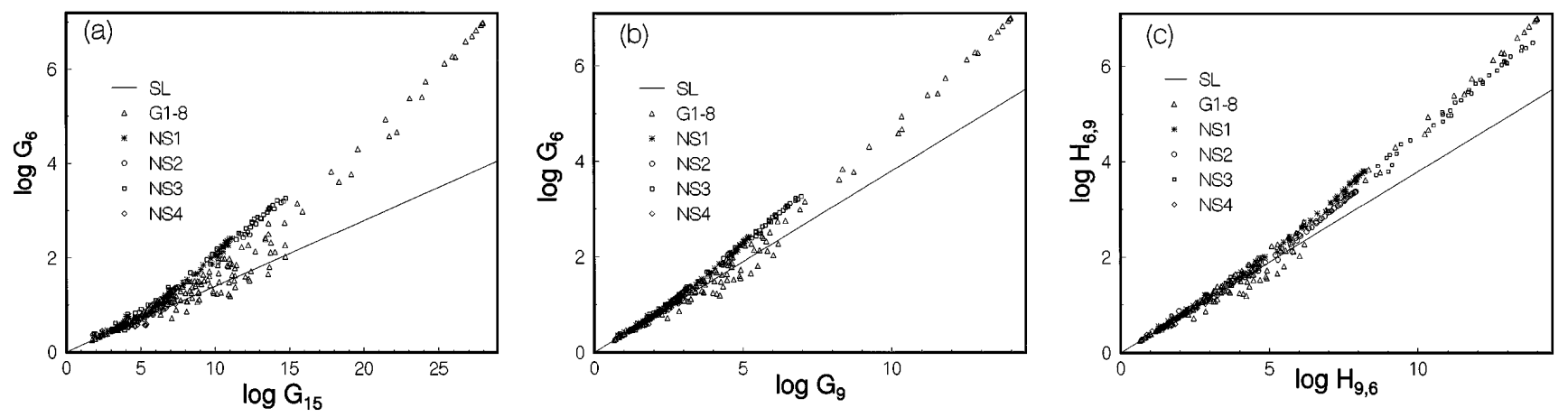

FIG. 3. Log-log (base 10) plots of $G_{6}$ versus (a) $G_{15}$ and (b) $G_{9}$ illustrating our $k$-space GESS; (c) $H_{6,9}$ versus $H_{9,6}$ showing the universal inertial- to dissipation-range crossover (see text). The line shows the SL, inertial-range prediction. 


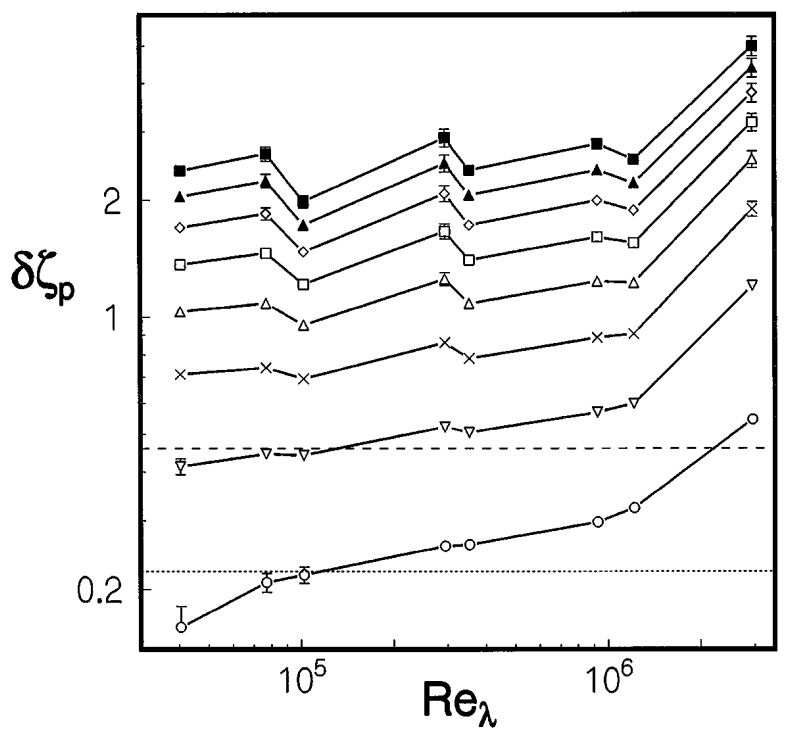

FIG. 4. Log-log plot (base 10) of $\delta \zeta_{p}$ versus the Taylormicroscale Reynolds number $\operatorname{Re}_{\lambda}$ for our GOY runs (G1-8) with $p=6,8 \ldots, 20$ (from bottom to top). The dotted ( $p=6$ ) and dashed $(p=8)$ lines show the SL results [4]. Error bars are shown but are often smaller than the symbol sizes.

Experimental evidence for the slope change in the dissipation range in real-space analogs of Fig. 1 was given by Stolovitzky and Sreenivasan [6], who postulated $S_{p} \sim S_{3}^{\alpha_{p}^{\prime}}$ in the dissipation range and suggested $\alpha_{p}^{\prime} \simeq$ $\left(\zeta_{3 p / 2}+p / 2\right) /\left(\zeta_{9 / 2}+3 / 2\right)$. We have not been able to obtain a simple relation between our $\alpha_{p}$ and their $\alpha_{p}^{\prime}$ (unlike [13] that between $\zeta_{p}$ and $\zeta_{p}^{\prime}$ ) since $S_{p}$ does not have a power-law dependence on $k$ in the dissipation range.

In conclusion, then, we have used our $k$-space ESS and GESS to obtain universal inertial-to-dissipation-range crossover in structure functions. It would be interesting to test this novel universality of dissipation-range asymptotics in diferent flows. The multiscaling we find in the far dissipation range might, at first sight, seem surprising because dissipation dominates here, but, as has been noted earlier [15], the intermittency seen in the far dissipation range can plausibly enhance mean nonlinear transfer even at low $\operatorname{Re}_{\lambda}$. Our dissipation-range multiscaling is a manifestation of such intermittency. Preliminary studies [26] yield similar phenomena in MHD turbulence.

We thank S. Ramaswamy for discussions, CSIR and BRNS (India) for support, and SERC (IISc, Bangalore) for computational resources.

*Also at Jawaharlal Nehru Centre for Advanced Scientific Research, Bangalore, India.

[1] A. N. Kolmogorov, C. R. Acad. Sci. USSR 30, 301 (1941).

[2] R. Benzi, L. Biferale, S. Ciliberto, M. Struglia, and R. Tripiccione, Europhys. Lett. 32, 709 (1995).

[3] F. Anselmet, Y. Gagne, E. J. Hopfinger, and R. A. Antonia, J. Fluid Mech. 140, 63 (1984).
[4] Z. S. She and E. Leveque, Phys. Rev. Lett. 72, 336 (1994).

[5] R. Benzi, S. Ciliberto, R. Trippiccione, C. Baudet, F. Massaioli, and S. Succi, Phys. Rev. E 48, R29 (1993).

[6] G. Stolovitzky and K. R. Sreenivasan, Phys. Rev. E 48, R33 (1993).

[7] M. Meneguzzi and A. Vincent, in Advances in Turbulence 3, edited by A. V. Johansson and P. H. Alfredsson (Springer, Berlin, 1991), pp. 211-220.

[8] J. Herweijer and W. van de Water, Phys. Rev. Lett. 74, 4651 (1995).

[9] M. H. Jensen, G. Paladin, and A. Vulpiani, Phys. Rev. A 43, 798 (1991).

[10] D. Pisarenko, L. Bieferale, D. Courvoisier, U. Frisch, and M. Vergassola, Phys. Fluids A 5, 2533 (1993).

[11] L. Kadanoff, D. Lohse, J. Wang, and R. Benzi, Phys. Fluids 7, 617 (1995).

[12] E. B. Gledzer, Sov. Phys. Dokl. 18, 216 (1973); K. Ohkitani and M. Yamada, Prog. Theor. Phys. 81, 329 (1989).

[13] This result is new. Our NS runs, though restricted to relatively low $\operatorname{Re}_{\lambda}(\$ 22)$, uncover it via ESS and $\zeta_{3}=1$. For $p$ even this result follows via dimensional analysis if one Fourier transforms the real-space $S_{p}$ and makes the numerically plausible assumption that $\left\langle\mathbf{v}_{i 1}\left(\mathbf{k}_{\mathbf{1}}\right), \ldots, \mathbf{v}_{i p}\left(\mathbf{k}_{\mathbf{p}}\right)\right\rangle$ is dominated by terms in which the $\mathbf{k}_{m}, m=1, \ldots, p$ arguments form equal and opposite pairs all with magnitude $k$, i.e., $\left\langle\mathbf{v}_{i 1}\left(\mathbf{k}_{\mathbf{1}}\right), \ldots, \mathbf{v}_{i p}\left(\mathbf{k}_{\mathbf{p}}\right)\right\rangle \sim$ $S_{p}(k)\left[\delta\left(\mathbf{k}_{1}+\mathbf{k}_{2}\right) \cdots \delta\left(\mathbf{k}_{p-1}+\mathbf{k}_{p}\right)+\right.$ permutations $]$. Other authors [27] make this assumption, but use further approximations to obtain different results.

[14] With $-\nu_{H} k^{\alpha_{H}}$ dissipation, $\zeta_{p}$ depend on $\alpha_{H}$; this nonuniversality is removed in plots like Fig. 3 , at least in the inertial range [E. Leveque and Z. S. She, Phys. Rev. Lett. 75, 2690 (1995); V. Borue and S. A. Orszag, Europhys. Lett. 29, 6875 (1995)]. With our $-\left(\nu k^{2}+\nu_{H} k^{\alpha_{H}}\right)$ dissipation, $\nu \neq 0$ and $2<\alpha_{H}$, so $\nu$, not $\nu_{H}$, controls $\zeta_{p}$.

[15] S. Chen, G. Doolen, J. R. Herring, R. H. Kraichnan, S. A. Orszag, and Z.S. She, Phys. Rev. Lett. 70, 3051 (1993).

[16] C. M. Bender and S. A. Orszag, Advanced Mathematical Methods for Scientists and Engineers (McGraw-Hill, New York, 1978), p. 80.

[17] T. Katsuyama, Y. Horiuchi, and K. Nagata, Phys. Rev. E 49, 4052 (1994).

[18] S. Grossman, D. Lohse, V. L'vov, and I. Procaccia, Phys. Rev. Lett. 73, 432 (1994).

[19] V.S. L'vov and I. Procaccia, Phys. Rev. Lett. 74, 2690 (1995).

[20] S. Chen, G. D. Doolen, R. H. Kraichnan, and L -P. Wang, Phys. Rev. Lett. 74, 1755 (1995).

[21] The increase in $\delta \zeta_{p}$ for run G8 did not go away on reducing $\delta t$ to $4 \times 10^{-6}$, with $\tau_{t} \simeq 500 \tau_{e}$ and $\tau_{\mathrm{av}} \simeq$ $2500 \tau_{e}$.

[22] In the dissipation range, $G_{p} \sim k^{\beta_{p}^{\prime}} \exp \left(-c_{p}^{\prime} k / k_{d}\right)$, so there is no SL analog for $\alpha_{p}$.

[23] This increases the inertial range by $2-3$ octaves relative to forcing the fourth shell $[10,11]$.

[24] $\Sigma_{n, p}$ yields a slightly lower estimate for $\zeta_{p}$ than $S_{n, p}$.

[25] D. Lohse, Phys. Rev. Lett. 73, 3223 (1994).

[26] A. Basu, S. K. Dhar, A. Sain, and R. Pandit (unpublished).

[27] V.S. L'vov, Phys. Rep. 207, 1 (1991); V.S. L'vov and I. Procaccia, Phys. Rev. E 49, 4044 (1994). 